\title{
La forma come funzione della grandezza.
}

Ricerche sul sistema muscolare degli Invertebrati

del

Dottor Guido Grandi

(Bologna, Istituto Zoologico).

Colle Tavole XII e XIII.

Eingegangen am 27. Oktober 1911.

Sommario.

I. Introduzione . . . . . . . . . . . . . . . . . . . . . 239

II. Tecnica . . . . . . . . . . . . . . . . . . . . . . . . . . . . 241

III. Vermi

1. Trematodi Malacocotili (Distomidae) . . . . . . . . . . . . 242

2. Nematodi prop. detti . . . . . . . . . . . . . . . . . . . . 244

3. Oligocheti terricoli (Lombricidae) . . . . . . . . . . . . . . . . 244

4. Sipunculidi . . . . . . . . . . . . . . . . . . . . . . . . . . 249

IV. Molluschi

1. Gasteropodi polmonati. . . . . . . . . . . . . . . . . 250

2. Cefalopodi (Ottopodi) . . . . . . . . . . . . . . . . . . . . . 253

3. Cefalopodi (Decapodi) . . . . . . . . . . . . . . . . . . 257

V. Conclusione . . . . . . . . . . . . . . . . . 258

VI. Zusammenfassung . . . . . . . . . . . . . . . . . . . . . . . . 259

VII. Bibliografia . . . . . . . . . . . . . . . . . . . . . . . 260

VIII. Spiegazione delle figure. . . . . . . . . . . . . . . . . 261

\section{Introduzione.}

Il Prof. Paolo Enriques ha iniziato con due memorie uno studio - per decidere quale influenza abbia la grandezza sui vari sistemi di organi nelle specie diverse $<$; la prima di queste memorie tratta l'economia di sostanza delle ossa cave, la seconda si occupa dei gangli nervosi degli Invertebrati. Mio scopo è stato di estendere queste ricerche al sistema muscolare di alcuni Invertebrati.

È noto che un organo può modificarsi per l'uso, e che anzi un uso prolungato può trasformarlo, se è di forza piccola o mediocre, 
in uno di forza, e, spesso e visibilmente, anche di volume maggiore. È pure ben noto che l'azione dell' uso si fonda sul fatto che all' organo corrispondente sono portati di continuo degli stimoli, conoscinti col nome di stimoli trofici, i quali si cambiano in attività; e che quest' azione non agisce solo grossolanamente aumentando o diminuendo la forza ed il volume, ma bensì modificando le più minute conformazioni costruttive.

Appare dunque subito, a priori, come essendo i muscoli elementi molto variabili, in causa della grande loro suscettibilità a questi stimoli, difficoltosa resulti un' osservazione, la quale sappia convenientemente trascurare tatto ciò che nelle parti esaminate dipenda da cause modificatrici particolari.

Individui sistematicamente molto affini conducono spesso vita affatto diversa, e il sistema muscolare può di conseguenza essere sviluppato da attività notevolmente varie e presentarsi diversamente costituito in organi omologhi, indipendentemente dal maggior o minor grado evolutivo raggiunto dalla" specie.

Risulterebbe infatti evidentemente artificioso (per il nostro scopo) un paragone fra i muscoli dell' avambraccio di una talpa e quelli di nn toporagno; fra quelli della tibia anteriore di un grillo e quelli di una grillotalpa, giacchè in ambedue $i$ casi lo sviluppo maggiore dei secondi rispetto ai primi non sarebbe in funzione della grandezza, e si prenderebbero così in considerazione canse modificatrici di effetto particolare trascurando quelle generali.

Queste diverse funzioni di organi omologhi, note e ben evidenti in alcune specie, sono incerte, oscure e affatto sconoscinte in altre; nel nostro caso adunque, oltre che attenerci a confronti comparativi fra specie dello stesso genere o di generi affini, ci si è dovuti limitare allo studio dei muscoli di quelle parti, le quali, nel limite del possibile, ci dessero fidanza della massima affinità funzionale.

Dagli studi di EnRIQues e dai nostri è apparso che l'organizzazione delle specie si complica in ragione diretta della loro grandezza. Ora noi vorremmo intenderci brevemente sul significato di questa > complicazioner. Complicazione nel caso nostro particolare, noi intendiamo per costituzione (architettonica ed istologica) atta ad offrire una resistenza, e quindi una forza, proporzionata all' aumento di volume dell' individuo. Ma resistenza maggiore verso un qualsiasi fenomeno esterno noi possiamo ottenere sia rinforzando maggiormente le singole parti costitutive, sia aumentando il loro numero e distribuendole in 
modo opportuno. 亡 sotto queste due forme che noi vedremo, nel corso delle presenti ricerche, presentarsi la costituzione architettonica muscolare delle singole specie.

Vogliamo subito rendere noto però (perchè male o confusamente non venga interpretato il significato e lo scopo di questo studio) che non si è inteso di fare confronti fra l'organizzazione di un gruppo o di un ordine con quella di un altro gruppo e di un altro ordine, ma soltanto fra le specie di ciascun gruppo, sicchè $i$ resultati valgono appunto singolarmente per ognuno di essi.

\section{Tecnica.}

Riusciti secondo le nostre possibilità ad eliminare, o meglio a scartare, tutto ciò che nella costitnzione stessa dell' individuo potesse male avviare lo studio, è stato necessario seguire una tecnica tale da impedire un rigonfiamento od una restrizione artificiale dei muscoli. Particolarmente indaginoso si è presentato il trattamento dei Molluschi polmonati. Per gli Stilommatofori si è dovuto scartare a priori la morte in acqua priva d'aria o con aria ed il taglio netto dei pezzi dall' animale vivo; i primi come mezzi fortemente dilatatori del sistema muscolare, i secondi come troppo restrittori. L'uso dell' olio freddo e caldo, dell' acqua alcoolizzata non hanno fatto miglior prova. Si sono invece ottenuti resnltati soddisfacenti immergendo i molluschi, fino alla morte, in un miscuglio così composto:

95 parti soluzione di $\mathrm{NaCl}$ al $6 \%$,

5 - alcool a $90^{\circ}$.

I Basommatofori, lasciati nella loro acqua, sono stati posti in recipiente chiuso, ed in esso, mediante un apparecchio di KIPP, si è immesso lentamente $\mathrm{Co}_{2}$. Di tutti i Cefalopodi, e della maggior parte dei Vermi, si è fatto dissezione anatomica previo trattamento con cloroformio. Quando degli Oligocheti terricoli si sono volnte intere sezioni trasversali, per studiare la topografia muscolare, si è fatto evacuare il terriccio, lasciando per qualche tempo $i$ vermi viyi in polvere di caffè umida, la quale, sostituendo in breve quello nell' intestino, permette il taglio senza troppi inconvenienti. Del resto la maggior parte di essa è stata pure eliminata mediante l'azione sull' animale dei due reofori di una piccola pila.

La fissazione del materiale si è ottenata immergendo i pezzi per 12-24 ore, secondo il loro volume, in una soluzione di: 


$$
\begin{array}{ll}
\mathrm{HgCl}_{2} & \mathbf{5} \mathrm{g}, \\
\mathrm{K}_{2} \mathrm{Cr}_{2} \mathrm{O}_{7} & 2,5 \mathrm{~g}, \\
\mathrm{H}_{2} \mathrm{O} & 100 \mathrm{~g} .
\end{array}
$$

Al momento della immersione la soluzione era riscaldata fino a $80^{\circ}$ centigradi; subito dopo tolta dalla fiamma e lasciata raffredare a poco a poco. Si è avuto cura, dopo la fissazione, di fare lunghi lavaggi ( $24-36$ ore) dei pezzi in acqua corrente, e molto lenti e graduali i passaggi negli alcool, nel toluolo e nelle paraffine.

Per la colorazione si è usato esclusivamente l'emallume e la fuxina acida; abbiamo colorato sempre in sezione e mai in toto. La grandissima parte dei pezzi è stata sezionata in serie, e di ognuno di essi abbiamo fatti tagli graduali da 5 a 25 micr. Alle ragioni che ci hanno fatto scegliere parti diverse nelle singole specie abbiamo già accennato più avanti, e di esse si dirà particolarmente nella descrizione comparativa.

\section{Vermi.}

1. Trematodi Malacocotili (Distomidae).

Abbiamo esaminato di questa famiglia le due specie Fasciola hepatica e Dicrocoelium lanceatum. La loro differenza in grandezza è sufficiente perchè si possa avere un' idea chiara della disposizione della musculatura nelle due forme e delle relative variazioni.

Si sono studiati particolarmente i muscoli che circondano, fasciandolo in certa maniera, tutto il corpo; e quelli che costituiscono le ventose e la faringe. In $F$. hepatica la zona di muscoli circondante il corpo è costituita da tre strati: un primo esterno, comunicante direttamente con quello a fibre elastiche che costituisce la sottocuticola, formato da fibre decorrenti circolarmente attorno al corpo del verme; uno immediatamente susseguente di fibre muscolari longitudinali, dirette secondo l'asse maggiore del corpo; ed infine un terzo strato ed ultimo di fibre a decorrenza obliqua. Con un po' di cura, si può, sezionando un soggetto trasversalmente, rendere visibile, specialmente nella parte anteriore (orale), contemporaneamente tutti e tre questi strati (fig. 2); ed allora appare chiaramente l'aspetto massiccio e complicato di questa musculatura. Ogni strato è costituito di fibre uniorientate.

La ventosa orale e quella ventrale sono costituite presso a poco similmente, accenneremo quindi solamente al tipo generale. La fig. 1 mostra schematizzata una sezione sagittale del polo orale di 
una $F$. hepatica; sono chiaramente visibili la ventosa orale e la faringe. Riportiamoci alla prima: una serie di fibre attraversa la sezione sagittale della ventosa percorrendola pel suo asse minore. Queste fibre ben distinte e costituenti da sole una gran parte della musenlatura della ventosa, sono le così dette fibre radiali. Tutto intorno alla sezione, lungo il margine di essa, si vedono sezioni trasverse di altre fibre che sono le equatoriali interne ed esterne. Finalmente decorrenti lungo l'asse maggiore, sempre della sezione sagittale, e incrociandolo, si osservano le fibre meridiane e quelle trasverse.

La costituzione istologica della faringe è molto simile a quella delle ventose. In gran numero, pressate molto le une alle altre occupanti quasi totalmente la massa faringea, sono le fibre muscolari radiali. Attorno ai margini della sezione, come si è osservato nelle ventose, si vedono i tagli trasversi delle fibre costituenti uno strato esterno di fibre circolari. Ne resulta un tessuto eminentemente massiccio ed omogeneo che ben riflette la forza relativamente poderosa di quest' organo. La faringe può essere proiettata in avanti nella cavità boccale (cavità della ventosa) e sucessivamente ritirata; questo movimento è reso possibile rispettivamente dal muscolo protrattore della faringe (fig. $1 \mathrm{mp}$ ), grosso muscolo che si inserisce colla sua larga base alla parte inferiore della ventosa, e col suo apice alla base della faringe; e dal muscolo retrattore della faringe, inserito ai tegumenti della regione orale e all' apice della faringe.

In $D$. lanceatum la tenue costituzione generale, e, in certo modo, la semplicità della musculatura (la specie è cinque o sei volte più piccola - dimensioni lineari - della precedente), ci dispensano da una descrizione particolareggiata. Tanto la zona di muscoli circondante il corpo, quanto quelle che costituiscono le ventose e la faringe, si mostrano estremamente lasse (fig. 3). Del resto questo $\grave{\mathrm{e}}$ fenomeno generale e ben evidente ad un esame superficiale. Se infatti si osservano due individui di queste singole specie, colorati in toto, immersi in un liquido chiarificatore e pressati semplicemente fra due retri, si vedranno in Dicrocoelium, per quasi totale trasparenza, molto nitidamente tutti gli organi interni; mentre di queste parti in Fasciola sono visibili solamente ed incerti i contorni.

La faringe p. d. che in Fasciola è lunga più del doppio della ventosa orale (sezione sagittale), in Dicrocoelium non raggiunge un terzo della grandezza lineare della stessa ventosa.

Nei Distomidi adunque noi vediamo che l'architettura muscolare subisce, in rapporto alla grandezza del corpo, un' evidente modifica- 
zione. Nelle specie di grossa mole $i$ muscoli in strati sovrapposti, con direzione unica in ogni singolo strato e ben complessi; nelle piccole specie ridotti a sottili e sparse fibre.

\section{Nematodi propriamente detti.}

Per quest' ordine non sono state necessarie ricerche speciali. $\mathrm{E}$ ben nota infatti la distribuzione degli elementi costruttivi muscolari nelle specie che lo costituiscono. Per brevità riferiremo senz' altro in proposito le parole del Prof. Emery tratte dal suo compendio di Zoologia:

- Nell' ordine dei Nematodi propr. detti le cellule muscolari hanno la loro parte contrattile rivolta all' esterno mentre la parte protoplasmatica, che racchiude il nucleo confina direttamente colla cavità viscerale. Quando si esamina nna sezione trasversale, si riconosce che quattro ispessimenti dell' ipodermide, i quali costituiscono le linee mediane e laterali, dividono lo strato muscolare in due fasci dorsali e due ventrali. In generale nei piccoli Nematodi, che diconsi perciò meromiarii, le cellule muscolari che vengono incontrate in una sezione sono poche e le loro fibrille contrattili formano uno strato piatto sotto l'ipodermide, onde il nome di platimiarii (Oxyuris); nelle grandi specie il numero delle cellule muscolari incontrate nella sezione supera il numero di otto; diconsi perciò polimiarii e lo strato di fibrille contrattili suole ripiegarsi su di sè stesso in ciascuna cellula come nell' Ascaride; nello stesso verme la parte protoplasmatica delle cellale muscolari che contiene il nucleo, costituisce appendici sporgenti nella cavità viscerale per cui questa forma di musculatura è detta celomiarias.

Nei Nematodi dunque le specie di grossa mole si mostrano, riguardo ai muscoli, più complesse ed in maniera tale che su questa complicazione si è potata basare una fondata distinzione.

\section{Oligocheti Terricoli (Lombricidae).}

Le specie di quest' ordine scelte per il nostro studio appartengono ai generi Octolasium ed Eiseniella e sono: l'O. complanatum Ant. Dug. che è una delle forme indigene più grosse dell' ordine intero; e l'E. tetraedra rispettivamente una dèlle più piccole. Ambedue essendo comuni nel fango umido delle nostre pianure, abbiamo potato avere a nostra disposizione un abbondante materiale 1 ).

1) La determinazione di queste specie è stata fatta dal Dott. Lurgi Cognetri di Torino, al quale rivolgiamo qui vivi ringraziamenti. 
L'anatomia dei Lombricidi, almeno per quanto riguarda la topografia generale, è ben conosciuta; questo faciliterà il nostro compito, e ci permetterà di discutere fatti e costituzioni facilmente controllabili.

Nella sezione trasversa della parte media del corpo di un $O$. complanatum (fig. 5), procedendo dall' esterno verso l'interno, si distingue ehiaramente dopo la membrana molto sottile che costitaisce la cuticola di rivestimento, uno strato di cellule non ben distinte, coll' asse maggiore perpendicolare alla cuticola, irregolarmente cilindriche, slargate all' estremità. Queste cellule costituiscono l'epiderma. A questo strato epidermico ne segue uno mnscolare, il quale normalmente ha uno spessore doppio del primo; ma in molti punti quadruplo; in ogni caso mai minore del doppio. É costituito da fibre decorrenti circolarmente e ricchissimo di pigmento giallo sparso qua e là molto irregolarmente. Come terzo strato dopo la cuticola ne dobbiamo menzionare uno ben caratteristico, quello cioè costituito dai muscoli longitudinali penniformi. La costituzione di tali muscoli, dopo la deserizione del Clapark̀de, è ben conosciuta. Si ha una sorta di lamella ${ }^{1}$ ) che serve di asse di sostegno, alla quale sono inserite obliquamente una grande quantità di lamelle secondarie, paragonabili agli assi delle barbule, e con estremità libere. La base delle lamelle principali, e quindi dei singoli elementi muscolari, poggia sul limite interno della regione dei muscoli circolari, mentre l'apice di esse si confonde col peritoneo. Questo strato ha sempre uno spessore quadruplo di quello a fibre circolari, talvolta anche quintuplo, ma non è continuo, essendo interrotto ai punti d'inserzione delle setole, e risultando cosi definitivamente costituito di una serie di zone dorsali, laterali, ventrali ed accessorie. Accessorie sono quelle comprese fra le due diramazioni di una setola.

In una sezione sagittale della medesima parte, lo strato muscolare circolare si presenta coi fasci in sezione trasversa; sezioni di fasci che, essendo ben pressate fra loro, spiegano una compattezza ben decisa dei fasci stessi.

In E. tetraedra, e in sezioni simili a quelle vedute per Octolasium, i diversi strati si susseguono collo stesso ordine, ma variano in estensione (fig. 4). La zona epidermiea è molto alta; quella a muscoli circolari invece è molto ridotta; di solito rappresenta la

1) SCHNEIDER chiama piccole cassette muscolari Muskelkästchen \& gli spazi limitati da queste lamelle principali. 


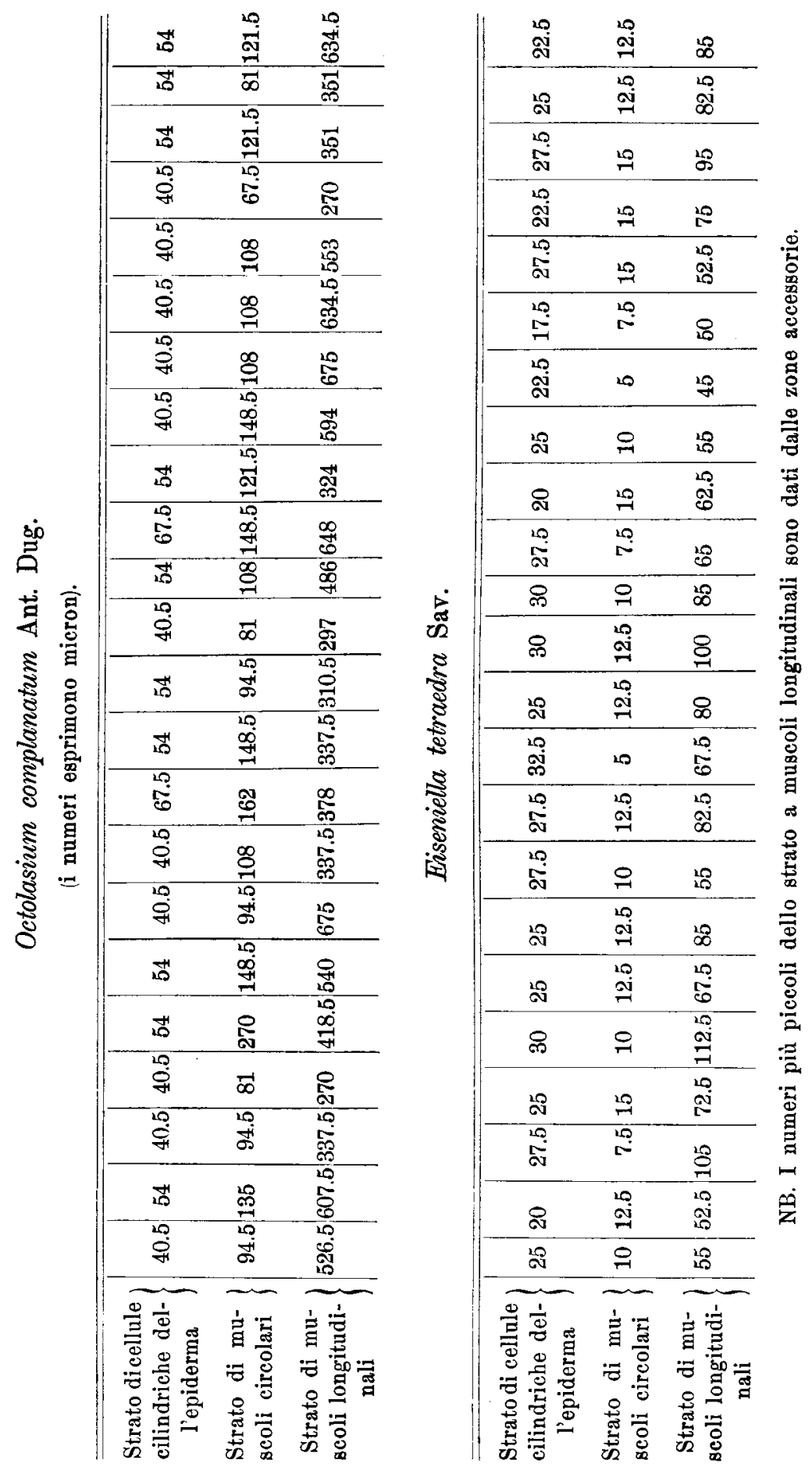




\begin{tabular}{|c|c|c|}
\hline \multirow{3}{*}{$\sigma$} & 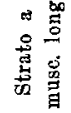 & 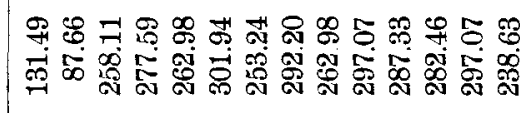 \\
\hline & 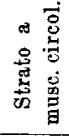 & 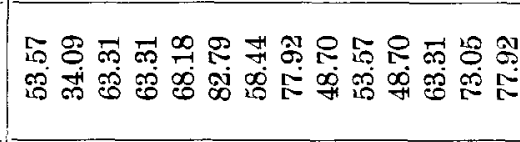 \\
\hline & 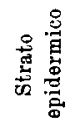 & 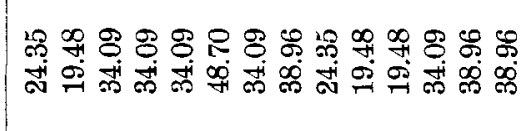 \\
\hline \multirow{3}{*}{0} & 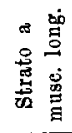 & 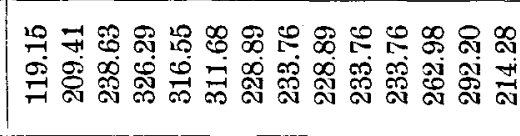 \\
\hline & 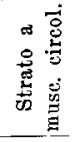 & 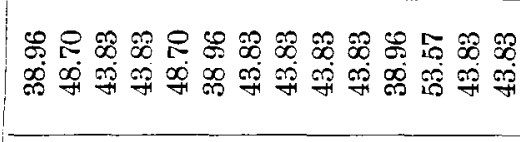 \\
\hline & 总泀 & 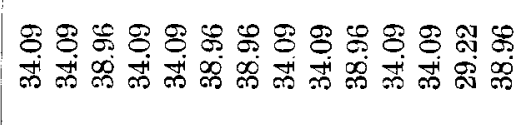 \\
\hline \multirow{3}{*}{ م } & 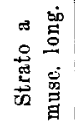 & 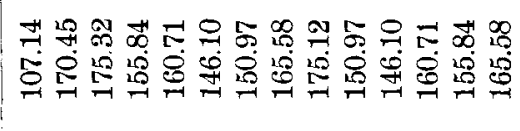 \\
\hline & 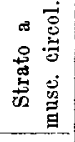 & 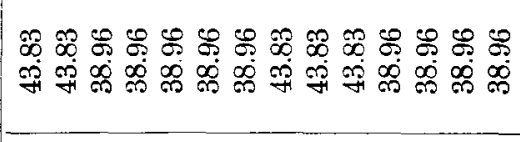 \\
\hline & 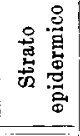 & 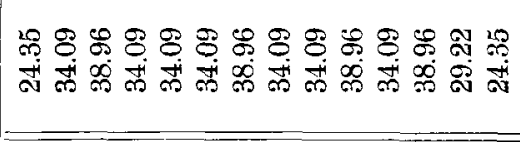 \\
\hline \multirow{3}{*}{ +3 } & 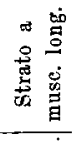 & 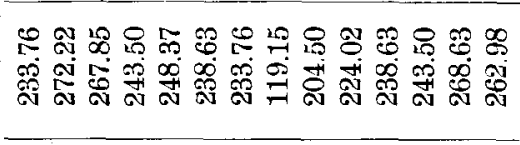 \\
\hline & 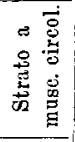 & 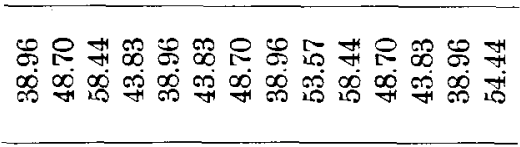 \\
\hline & की & 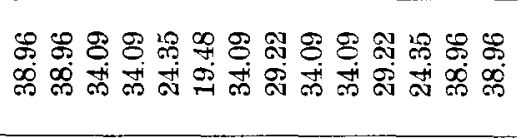 \\
\hline \multicolumn{2}{|c|}{ 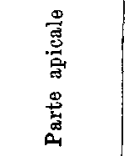 } & 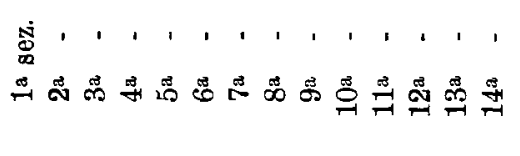 \\
\hline
\end{tabular}

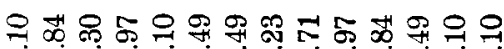

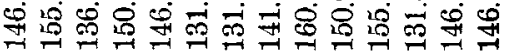

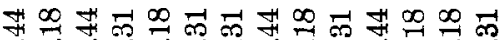
வ

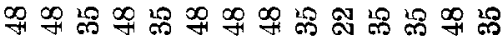

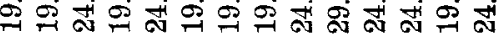

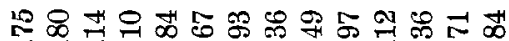

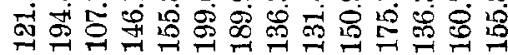

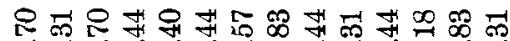

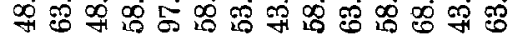

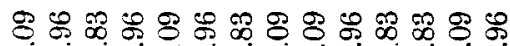
मां

농응 웅

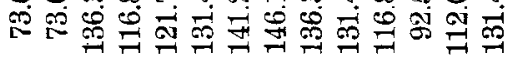

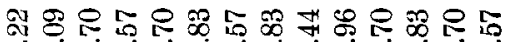

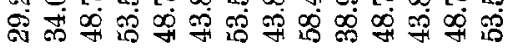

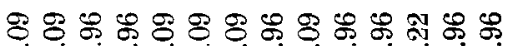

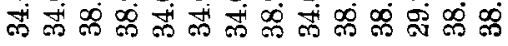

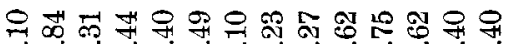

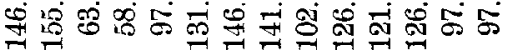

வิ

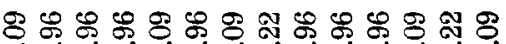

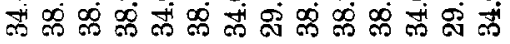

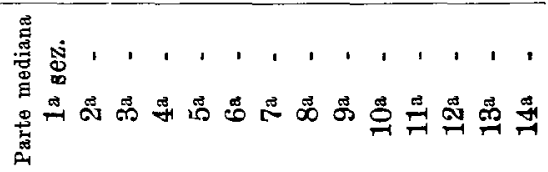


quarta parte della prima, talvolta la metà, non mai si mostra di maggior spessore. Lo strato a muscoli longitudinali, è supergiù ugualmente costituito.

Esaminando sezioni sagittali della medesima parte si vede come, pur rimanendo generalmente la stessa disposizione topografica, resulti evidentissima la grande riduzione dello strato a fibre circolari, ridotto qui, ad una sottile zona di sezioni trasverse di fibre, strato ben delineato nella fig. 6 .

Se ora diamo uno sguardo complessivo ai resultati ottenuti vediamo come nelle parti da noi studiate varii l'estensione delle zone costituenti. Una misurazione micromillimetrica accurata condotta in molte sezioni ci ha dato per Octolasium e per Eiseniella i dati esposti nella prima tabella. Il criterio statistico che ne resulta è ben chiaro. Considerando, naturalmente, come elementi varianti i muscoli e confrontando questi, ad esempio, collo strato di cellule cilindriche costituenti l'epiderma, si vede come nella grossa specie (Octolasium) lo strato di muscoli circolari sia di uno spessore doppio, triplo ed anche quadruplo di quello epidermico, mentre nella piccola specie (Eiseniella) queste proporzioni addiritura si invertono; in essa l'epiderma ha di solito un' altezza quattro o tre volte maggiore a quella della zona muscolare sottostante; talvolta, ma raramente, due volte solo. Di più si è voluto vedere se gli strati in discorso variassero reciprocamente di estensione nelle diverse parti del corpo dell' animale, dal momento che alcuni autori accennano ad una possibile variazione. La seconda tabella che accompagna questo lavoro ci dà, con sufficiente chiarezza, un' idea di come vadano le cose ${ }^{1}$ ). Naturalmente sui valori di questi numeri è bene intenderci.

Non si deve da essi detrarre una media complessiva, che, forzatamente, data la saltuarietà delle misure e delle sezioni, non avrebbe che un valore ben incerto, ma bensi osservando tutta la tavola, come se il nostro occhio scorresse i preparati al microscopio, si può vedere come si comportino queste zone muscolari. Allora, trascurando quei pochi numeri, che si scostano bruscamente dai valori comuni, numeri che indicano l'altezza di un punto anormale per il trattamento subito dalla tecnica, appare come il rapporto dei diversi strati nelle parti estrema e media, sia sensibilmente lo stesso.

1) Le misurazioni sono prese ai quattro punti estremi di ogni sezione. La prima parte della tabella rappresenta i valori detratti dalla regione apicale del corpo di un lombrico; la seconda parte quelli tolti dalla regione media. 


\section{Sipunculidi.}

Si è scelto la massa dei muscoli retrattori della proboscide. La differenza di grandezza delle due specie osservate: Sipunculus nudus e Phymosoma granulatum, è molto grande: il primo raggiunge $250 \mathrm{~mm}$. di lunghezza, il secondo non arriva ai $20 \mathrm{~mm}$.

Ma se noi esaminiamo attentamente il modo di comportarsi degli animali vivi e la loro costituzione interna, vediamo rispettivamente che i movimenti del Phymosoma (come ha già osservato ENriques) sono molto più attivi di quelli di Sipunculus, e che le due masse dei muscoli retrattori accennate, confrontate fra loro, ci mostrano una grandezza affatto proporzionata a quella totale degli animali. Se si volessero fare previsioni si dovrebbe necessariamente supporre per lo meno uno stesso grado di complicazione dei due complessi muscolari, se non concederne uno maggiore a quello del Phymosoma. Si vedrà invece come, nonostante la grande semplicità di questi organi, si mostri ben netta una costituzione del tutto inversa.

Nella sezione sagittale della massa costituita dai muscoli retrattori della proboscide di Sipunculus nudus (fig. 7) si presentano grosse fibre discretamente pressate le une alle altre riunite in gruppi più 0 meno grandi. La costituzione in generale è molto semplice, le fibre non s'incrociano e non presentano grandi variazioni di spessore e di lunghezza. Le sezione trasversali (fig. 8) dei medesimi muscoli danno un' idea più chiara della distribuzione topografica delle fibre. Esse, infatti, si notano riunite in gruppi di due, tre, quattro e cinque piccole aree, ovali rotondeggianti, elissoidi, reniformi. Questi gruppi alla loro volta mostrano una tendenza molto spiccata ed evidente a riunirsi in gruppi di ordine superiore.

In una sezione sagittale dei muscoli della proboscide di Phymosoma granulatum (fig. 9) la costituzione appare sullo stesso piano semplice di quella di Sipunculus. Le fibre però non sono unite, ma molto meno pressate fra loro; questa maggiore lassità costitutiva è ben evidente in numerosi tagli che interessano le parti superficiali e profonde.

L'esame delle sezioni trasversali (fig. 10) conferma e rinforza il giudizio precedente. Si vedono distintamente le sezioni delle fibre rotondeggianti od ovali distribuite molto regolarmente; non si notano aggruppamenti nè accenni di aggruppamenti, come si è visto in Sipunculus, di più è evidente una maggiore e costante distanza fra le singole fibre. 
Se confrontiamo la costituzione dei muscoli di questo verme con quella del Sipunculus sopra descritto, la differenza ci appare molto chiara, e tanto più interessante, inquantochè la struttura molto semplice di queste masse muscolari e la loro reciproca non grande differenza di lunghezza, di superficie e di volume, paragonata a quella molto forte dell' intero corpo degli animali, non ci avrebbe fatto supporre a priori, e logicamente, un resultato così inaspettato, e del resto cosi confortante la nostra tesi. Dunque la massa dei muscoli retrattori della proboscide è nel Sipunculus più complessa che non nel Phymosoma.

\section{Molluschi.}

\section{Gasteropodi polmonati.}

I Polmonati terrestri si presentano come un gruppo molto adatto per le nostre ricerche. Costituendo un materiale di facile raccolta, ci offrono differenze ragguardevoli di grandezza nelle specie di uno stesso genere, ed inoltre una parte del loro corpo, il piede il quale, nel genere Helix ad esempio, ha ben sicuramente una medesima e costante funzione. Questi fatti sono per noi di somma importanza, per essi si tolgono cosi delle probabili cause di variazione, che potrebbero in qualche caso, renderci dubbiosi sul significato di questa o di quella costituzione. É evidente dunque che i resultati ottenuti studiando la fabrica dei loro muscoli, saranno di un interesse speciale, e però di essi si tratterà il più distesamente e minutamente possibile. La facilità suacennata di raccogliere questi animali ci ha permesso di fare confronti molto diligenti.

Le specie studiate sono Helix adspersa ed $H$. lucorum fra quelle di relativa gran mole; $H$. nemoralis ed $H$. variabilis fra quelle di piccola. Di ogni specie però si sono esaminati grandi e piccoli individui onde non ci sfuggissero, o fossero fraintesi i limiti di transizione.

\section{Helix adspersa e lucorum.}

La massa muscolare faringea è, nella sua generalità, ben complessa e resulta in gran parte costituita da numerosi e grossi fasci muscolari fortemente pressati gli uni agli altri; questo comportamento è ben visibile in tagli trasversi del corpo dell' animale, a livello della faringe, ed è riprodotto schematicamente nella fig. 16; in alcuni punti però esso si modifica per la presenza di nna sorta di tessuto a vacuoli poliedrici o rotondeggianti e di varia grandezza, 
che evidentemente $\dot{e}$ un tessuto ghiandolare, il quale inframezzandosi ai fasci muscolari determina un allontanamento reciproco dei fasci stessi, e così dà luogo a zone riportabili, con grande assomiglianza alla Fig. 17 disegnata pel piede. In sezioni più interne (rispettivamente dall' aspertura orale del mollusco all apice opposto) si nota che $i$ fasci muscolari vanno perdendo in molti punti la loro compattezza, e sono sostituiti spesso da semplici fibre pressate le une alle altre. Questa disposizione è rappresentata dalla fig. 14 e 19, che ne dà un' idea sufficiente.

Il piede offre una grande assomiglianza di disposizione e di costituzione muscolare col faringe; si ha in esso però una più ampia estensione ed un maggior sviluppo del tessuto ghiandolare, ed in molti punti, un comportamento assai più semplice. Studieremo successivamente diversi tagli susseguenti e vedremo come vadano minutamente le cose. Iniziamo l'esame dell' apice orale: Fibre isolate od aggruppate irregolarmente in piccolo numero, per lo più dirette nel verso del piede, ma spesso tagliate od incrociate da altre trasversali; poi fibre isolate $o$ riunite in vario numero orientate in tatte le direzioni, pochi ed isolati vacuoli; procedendo: tessuto ricchissimo di grandi e piccoli vacuoli, fra essi fibre riunite in fascetti, raramente isolate, decorrenti per lo più longitudinalmente; più oltre ancora $i$ vacuoli vanno diradandosi ed isolandosi, le fibre sono pressochè tntte riunite in grossi fasci; finalmente $i$ vacuoli scompaiono del tntto, $i$ fasci si pressano sempre più ed acquistano un aspetto molto massiccio. Partiamo ora dal lato del piede strisciante sul suolo e procediamo in alto ed internamente: Tessuto costituito dai soliti fasci incrociantesi e diretti per tutti i versi; in alcuni punti il loro aspetto è lasso, i tagli trasversi di fibre aggruppati o no sono abbondantissimi; in altri i fascetti sono più numerosi e comincia ad individualizzarsi qualcuno dei soliti grandi fasci che percorrono longitudinalmente e per lungo tratto il pezzo, pochi $i$ vacuoli, molti i tagli di fibre trasverse aggruppati. Le costituzioni più complesse occupano gradualmente la zona superiore delle sezioni, corrispondente a quella anteriore del piede dell' animale; $i$ lati delle medesime, riferibili pure ai lati di questo organo muscolare, sono caratteristiche perchè ricche di numerosi vacuoli, qua e là inframezzati da lunghi e gràcili fasci. Proseguiamo: Va man mano perdendosi la costituzione lassa dei fasci; questi si mostrano via via più complessi, aumenta il numero dei gruppetti dei tagli trasversi di fibre e quello dei fasci lunghi. Altre sezioni, e la massiccità del tessuto si accresce visibilmente. Si nota in molte 
parti un comportamento speciale costituito da numerosi piccoli vacuoli framischiati ai fasci; ai lati il solito tessuto ghiandolare; infine la complessità ragginnge il massimo suo sviluppo, i fasci, in gran parte molto fitti, sono diretti più o meno obliquamente e tagliati trasversalmente, o con diversi angoli più o meno ampi, da altri di ugual spessore.

L'architettura muscolare delle parti esaminate è principalmente caratterizzata dalla presenza di grossi fasci, i quali, sia procedendo paralleli gli uni agli altri, sia incrociandosi ed aggrovigliandosi più o meno strettamente, danno sempre al complesso un aspetto poderoso e massiccio. In molti punti veramente questa massiccità diminuisce, in altri quasi scompare; rispettivamente presentandosi fasei meno forti, di costituzione più lassa, maggiormente distanziati fra loro, e fibre isolate; pur tuttavia l'estensione, e sopratutto la locazione di queste zone non è tale che possa influire menomamente su quanto si è detto più sopra, nè togliere, per il nostro scopo, importanza ad una costituzione cosi nettamente caratteristica, e così facilmente rintracciabile anche ad un esame superficiale di una sezione qualsiasi.

\section{Helix nemoralis e variabilis.}

Le piccole specie di Helix presentano, nella musculatura del faringe e del piede, un tessuto resultante di fibre isolate ed in buon numero; nelle zone di massimo sviluppo le fibre crescono di numero, diventano più fitte, si orientano in una stessa direzione; in quelle di minimo si diradano e si orientano in diverse direzioni.

Nell' H. nemoralis i muscoli faringei sono specialmente noteroli per la loro costituzione lassa e resa caratteristica dalla distribuzione uniforme di fibre sempre isolate, multiorientate $e$, in conseguenza, incrociate le une colle altre. La fig. 18 che li rappresenta in una sezione trasversa del faringe, è molto esplicativa a questo riguardo. I tagli trasversali delle fibre, irregolarmente sparsi qua e là, poco numerosi, ci fanno supporre che un identico comportamento si presenti nelle sezioni sagittali: Il loro esame conferma. Questo è l'andamento quasi costante della muscolatura del faringe, e anche di quella del piede (fig. 21) di queste Helix. In qualche caso un aumento del numero delle fibre, e una conseguente loro intima compressione, determina una costituzione un po' più complicata che può ritenersi come massima per questo gruppo di piccole specie (v. fig. 20).

Concludendo per i Polmonati stilommatofori: Dalla minuta esposizione dei caratteri osservati nelle diverse sezioni di questi molluschi 
appare chiaramente come aumenti gradatamente la complicazione e la massiccità degli elementi costruttivi del sistema muscolare, via via si sale dagli individai di piccola mole a quelli di mole maggiore. Se si osservano i due estremi di questa scala, si vede come la loro differenza sia enorme: Tessuto lasso, di aspetto estremamente semplice, con poche fibre isolate od unite in piccolo numero nei primi; fasci poderosi e compatti, ramificati, fluenti, aggrovigliati strettamente in ogni modo nei secondi. È utile occuparci un po' diffusamente degli elementi più o meno variabili. Passaggi fra l'una e l'altra forma ve ne sono e parecehi, ma non sono mai tali e di tale natura che possano assolntamente unire con insensibili variazioni i due tipi descritti.

Dal tessuto di estrema lassità ricordato come proprio della forma piccola, si passa ad un secondo grado di organizzazione dove unico elemento variante è il numero delle fibre; poi queste fibre, ancora aumentando, presentano qualche tendenza a perdere la loro disposizione sparsa, irregolare multiorientata e ad acquistarne invece una più regolare che le mostra dirette in un sol verso. In un grado ancor maggiore di questa loro evoluzione, si nota in esse facilità a raccogliersi in gruppi e regioni determinate, lasciando fra questi aggruppamenti spazi vaoti che sono occupati da vacuoli e da zone di fibre trasverse. Questo è l'ultimo limite di loro complessità. In forme piccole quali l' $H$. nemoralis e l' $H$. variabilis non si trova maggior complicanza nè fabrica più complessa di muscoli. Passaggi ho detto che esistono fra l'una e l'altra forma, ma è nel tipo grande che in qualche parte troviamo rappresentata la costituzione massima propria del piccolo. Così in H. adspersa ed in H. lucorum vediamo molti punti nei quali a fasci forti e ramificati e complessi sono uniti gruppi lassi di fibre isolate.

Dunque nelle specie piccole del genere Helix, in quelle parti da noi esaminate, il tessuto muscolare non raggiunge mai quel grado di complessa massiccità osservato nelle specie di maggior mole.

\section{Cefalopodi (Ott opodi).}

Le parti esaminate nelle specie di questo, e del sottordine seguente dei Decapodi, sono stati i bracci, come organi a funzione identica nelle forme, s'intende, proprie a ciascuno dei due sottordini.

\section{Eledone muscata.}

A piccolo ingrandimento la topografia generale della sezione trasversale del braccio (fig. 15) si presenta molto caratteristica: massic- 
eia e complicata, costituita di strati alternativamente longitudinali e trasversali, atta ad una massima resistenza.

Ad uno strato esterno di fibre decorrenti circolarmente rispetto all'osservatore, ne segue uno di fibre trasversali, attraversato da fasci di fibre longitudinali e perpendicolari a quelle del primo strato e del terzo; poi questo terzo simile al primo, ma più alto; un quarto di fibre trasversali riunite in gruppi, quasi tanto alto quanto gli altri tre riuniti insieme; e, come il secondo, attraversato da fasci di fibre molto grossi; finalmente un quinto ed ultimo che limita nel mezzo il foro centrale, costituito da fasci piuttosto lassi ed incrociantesi vicendevolmente.

Esaminiamo queste diverse parti più minutamente:

$1^{\circ}$ Strato di muscoli circolari (fig. 15, 1). Le fibre del primo strato sono riunite in un tutto compattissimo.

$1^{\circ}$ Strato di muscoli longitudinali (fig. 15, 2). Le sezioni trasverse delle fibre sono riunite in piccoli gruppi molto pressati gli uni agli altri e talvolta divisi da spazi irregolari. A distanze simili sono attraversati da fasci di fibre che si allargano ai punti di contatto col primo e col terzo strato, penetrano in questi a guisa di radici e, talvolta, li attraversano tutti.

$2^{\circ}$ Strato di muscoli circolari (fig. 15, 3). Il terzo strato è costituito fondamentalmente da fibre decorrenti in senso orizzontale e circolare, discretamente pressate fra loro; da fibre decorrenti in senso verticale, rappresentate dalla continuazione dei fasci trasversali del $2^{\circ}$ strato; ed infine da sezioni di fibre trasverse sparse qua e là.

$2^{\circ}$ Strato di muscoli longitudinali (fig. 15, 4). Grandi masse di sezioni trasverse di fibre riunite in gruppi distinti, e fasci decorrenti verticalmente.

$5^{\circ}$ Strato (fig. 15, 5). $\dot{\mathrm{E}}$ costituito dalla continuazione di questi fasci verticali e da altri che li tagliano quasi ad angolo retto.

Il quinto strato ha forma quadrangolare con due lati concavi verso l'esterno e due convessi, ma gli angoli si prolungano molto e raggiungono il terzo strato, dividendo cosi tutta la massa in quattro regioni. Le due laterali (dico laterali immaginando il pezzo orientato in maniera che la linea o le linee, a seconda che si tratta di Eledone o di Octopus, delle ventose si trovi in alto) presentano la costituzione tipica descritta; la dorsale, invece, e la ventrale sono diversamente conformate: In esse non si scorgono più i cinque strati 
caratteristici descritti sopra, ma solamente due, e cioè: il quinto identico a quello già studiato, ed un altro che assorbe il primo, il secondo, il terzo ed il quarto; alto, di consegnenza, come tutti questi insieme, e di costituzione molto simile a quella del quarto. Rappresentano queste regioni rispettivamente, la superiore i muscoli adduttori del braccio, l'inferiore quelli abduttori.

Se vogliamo ora renderci conto del comportamento generale di questa struttura, è necessario di assicurarci se essa si conserva tale in tatto l'organo esaminato e se su di essa può avere influenza lo spessore della sezione.

Un interno braccio tagliato ad intervalli dalla base fino al suo apice, non ci ha dato variazioni dei suoi elementi costruttivi, calcolabili in questo studio. L'osservazione dell' anatomia interna di uno dei bracci più piccoli dello stesso animale, ci ha portato ai medesimi resultati; la sua costituzione è paragonabile a quella della regione apicale di un' appendice di maggior volume, essendo in esso proporzionalmente ridotta la sola superficie.

\section{Ogtopus vulgaris ${ }^{1}$.}

Si mantiene la costituzione descritta per le grandi Eledoni, i fasci però acquistano una massicità ed una complicazione enorme: Sono vere e poderose colonne muscolari, che, partendosi con larga base dall' ultimo strato interno $\left(5^{\circ}\right)$ si innalzano, si ramificano e raggiungono il margine esterno. Gli angoli della zona quadrangolare costituita dai muscoli del quinto strato, non si risolvono qui subito in vari rami irradianti, ma si prolungano per lungo tratto. La complicazione intima muscolare dei bracci di questo individuo è tale, che tagli di $50 \mu$ non hanno dato sezioni a strati completi.

\section{Octopus macropus.}

La caratteristica lunghezza delle braccia di questa specie, non ha alcuna influenza sul comportamento muscolare interno. Per vero dire i bracci se sono molto lunghi (negli esemplari da me esaminati raggiungono i $750 \mathrm{~mm}$ ), non sono altrettanto larghi, e spesso, hanno il diametro della loro sezione trasversa minore di quello dell' Eledone; una sezione trasversale della base di uno dei bracci più grossi, ci si presenta poco diversa da quelle già esaminate nell' Eledone. Le fibre

1) Ci è pervenuto un braccio di questa specie, la cui sezione trasversa misurava $21 \mathrm{~mm}$. di diametro, per gentilezza del Dottor BaLdASSERoN che vivamente ringraziamo. 
decorrenti circolarmente all' esterno si mostrano forse divise in due o tre zone da setti sottilissimi; al di sotto del quarto strato, e prima del quinto, si nota uno strato simile al quarto, ma di altezza molto minore; di più gli spazi vuoti del quarto strato sono maggiormente estesi in superficie ${ }^{1}$ ).

\section{Octopus De-Filippii.}

Il tipo generale di struttura descritto per l'Eledone si mantiene anche qui (fig. 13). Il quinto strato però è quasi nullo, costituito di fibre non riunite in fasci e non incrociantesi come in quello. Le fibre decorrenti longitudinalmente all' estremo esterno non sono molto pressate; i fasci intersecanti sono esili talvolta filiformi. Le sezioni trasverse delle fibre hanno poca tendenza a riunirsi in gruppi, e spesso sono sparse qua e là irregolarmente. Anche il comportamento caratteristico dei poli delle sezioni si presenta con fasci molto ridotti, estremamente esili, e colle sezioni trasverse delle fibre molto più rade, distinte le une dalle altre e non riunite in piccoli campi. Se si esaminano in questa specie gli apici dei bracci e i bracci minori, si notano i soliti fenomeni di riduzione già descritti per l'Eledone e sui quali, naturalmente, è inutile ritornare.

Il confronto fra le specie esaminate di questo sottordine si presenta particolarmente interessante. Abbiamo, di fatto, potuto esaminare individui di gran mole, individui di piccola; e, di più, se così ci possiamo esprimere, individui in certo grado di transizione, ma di una transizione per molti riguardi interessante.

La differenza di costituzione fra l'Eledone muscata e l'O. DeFilippii, è molto evidente. In questo ultimo manca quasi lo strato alto e complesso, formato a guisa d'impalcatura, che abbiamo chiamato quinto interno. Gli strati tutti non sono compatti e massicci come nel primo. Le sezioni trasverse delle fibre, non sono riunite in campi od in zone determinate, ma sparse irregolarmente. I fasci che attraversano tutti questi strati, sono molto più esili e deboli e sottili. Il complesso infine presenta una topografia assolutamente pì̀ debolmente costituita, e nella quale gli elementi singoli sono disposti in modo da far giudicare molto minore la potenzialità della loro resistenza.

1) È da notarsi che il sacco degli O. macropus da noi esaminati era ben poco più grande di quello degli $O$. De-Filippii. Il primo non ragginngeva in media i $70 \mathrm{~mm}$; il secondo sorpassava i $50 \mathrm{~mm}$. 


\section{Cefalopodi (continuazione: Decapodi).}

Loligo vulgaris.

Grossi individui di questa specie con saceo di $170 \mathrm{~mm}$ di lunghezza e $50 \mathrm{~mm}$ di larghezza, e con bracci massimi lunghi più che $310 \mathrm{~mm}$, presentano, nelle sezioni trasverse di questi bracci una conformazione molto simile a quella degli Ottopodi. Anche qui strati diversi e concentrici (fig. 12). Il più esterno è formato di fibre ben pressate fra loro ed interrotte, ad intervalli quasi regolari, da sezioni trasversali di altre fibre, riunite in piccoli gruppi di tre o quattro sezioni. Questi gruppi occupano talvolta tutta l'altezza di questo primo strato; tal'altra si partono dal limite interno con larga base, ma non raggiungono quello esterno.

Il secondo strato è di fibre decorrenti circolarmente e molto fitte. Il terzo è occupato da sezioni trasverse di fibre riunite in numerosissimi piccoli campi. Questo strato è attraversato da fasci sottilissimi, sottili, mediocri e molto grossi, che non appare si continuino nel secondo strato descritto, ma si prolunghino invece nel quarto, il più esteso di tutti e percorso da una grande quantità di fibre molto fitte ed incrociate variamente.

Vogliamo ora vedere se la musculatura costituente le ventose, subisce essa pure qualche modificazione. In $L$. vulgaris se si esamina un taglio trasverso piuttosto profondo, sì da interessare possibilmente tatto il corpo della ventosa stessa, si nota subito nno strato esterno di fibre piuttosto fitte che decorrono verso l'interno. Dopo questo strato e fino quasi al foro centrale, tutta la superficie è occupata da sezioni trasverse di fibre riunite in campi più o meno estesi. Questa grande regione è separata a sua volta nettamente dal foro centrale, da una sorta di anello formata da fibre muscolari decorrenti circolarmente. A questo anello giungono, dopo avere attraversato la zona a fibre trasverse, numerosi fascetti muscolari pinttosto gracili, e quivi si espandono a guisa di radice. 亡̀ da notarsi che in qualche punto è ben visibile uno strato situato più esternamente a tutti quelli descritti, e costituito in modo simile a quello limitante il foro centrale.

Sepiola rondeletii e Loligo marmorae.

In sezioni del braccio, molto sottili di $7-8 \mu$ il foro centrale appare circondato da uno strato di fasci formato da fibre incrociate molto intimamente. Da questo partono e si irradiano dei fasei muscolari che raggiungono quasi il limite esterno, e che sono regolarmente 
separati da zone di sezioni trasverse di fibre, riunite nei soliti campi. Attorno ed all' esterno un sottile strato circolare di fibre trasverse (fig. 11). In sezioni di $15 \mu$ il tessuto appare naturalmente più compatto: I fasci muscolari che partono dallo strato interno e raggiungono l'esterno appaiono maggiormente sviluppati.

Anche la costituzione delle ventose è molto semplice in queste piccole specie: Tutta la zona, dal foro centrale al margine esterno, è occupata da fasci muscolari piuttosto esili che si assottigliano man mano si avvicinano al centro. Gli intervalli fra questi fasci sono occupati da tagli trasversi di fibre.

La differenza fra il tipo di costituzione delle specie piccole e quello delle specie grandi, è nei Decapodi ben distinta. Vediamo infatti in $L$. vulgaris la sezione di braccio con una complicazione di quattro strati successivi e diversi, con elementi costruttivi disposti in maniera, che vicendevolmente quasi si sostengano e si completino. In $S$. rondeletii ed in $L$. marmorae, uno strato circolare di muscoli attorno al foro centrale, dei fasci irradianti ed un esile strato esterno di fibre decorrenti circolarmente. La costitazione muscolare delle ventose presenta fra le varie forme studiate differenze simili, ed è molto più complicata in $L$. vulgaris.

Abbiamo così dato una scorsa alla costituzione dei bracci nei Cefalopodi, insistendo sui punti per noi particolarmente interessanti, ed abbiamo visto che la disposizione dei materiali costruttivi, e gli elementi stessi individualmente, si presentano più complicati nelle specie maggiori.

\section{v. Conclusioni.}

$\mathrm{Nel}$ succedersi progressivo delle osservazioni che siamo venuti facendo nel corso di queste ricerche, è apparsa in linea generale una complicazione della massa muscolare delle specie di grossa mole rispetto a quelle di mole media o piccola.

$\grave{\mathrm{E}}$ apparso pure, ben determinato nelle conclusioni particolari di ogni ordine esaminato, come questa maggiore o minore complessità sia talvolta ben chiara, sì che subito ne resulta una nitida distinzione fra i due tipi, tal' altra, pur rimanendo sempre calcolabile ed apprezzabile, un po' meno definita. Ma qui è opportuno osservare che noi ci troviamo in un campo di ricerche ben più ampio e variato di quello in cui si è trovato Enriques studiando i gangli nervosi degli Invertebrati; ed è facile persuadersi della difficoltà di un' orientazione, in seno ad un tessuto che per il suo stesso grande sviluppo 
si presenta così atto ad adattamenti vari, e quindi a variazioni assai complicate.

La struttura caratteristica dei Nematodi, nei quali è possibile definire la differenza di costitnzione fra le specie piccole (Meromiari) e quelle grandi (Polimiari) dal numero delle cellule muscolari che si incontrano in una sezione trasversale del verme, ci esprime, in una forma suscettibile di controllo matematico, il primo grado di complicazione che noi abbiamo trovato; rappresentato da un accrescimento di numero nei singoli individui muscolari.

In Sipunculus rispetto a Phymosoma la complicazione è leggermente progredita, essendosi gli elementi muscolari, cresciuti di numero, distribuiti a gruppi di due, tre e quattro elementi. Nelle grosse specie di Polmonati e di Dibranchiati si presentano già fasci muscolari ben individualizzati, in confronto col tessuto relativamente lasso delle forme piccole. Infine nei grandi Oligocheti fasci e fibre danno lnogo ad intere zone muscolari, a limiti netti e definibili nella loro superficie, zone che nelle specie di minor mole dello stesso ordine si mostrano mal definite, meno estese ed assai più debolmente e lassamente costituite.

Accertata dunque, pel sistema muscolare, la presenza d'una complicazione della sua struttura in ragione diretta della grandezza della specie, restano pure determinati, in linea generale, i vari modi coi quali questa complicazione si presenta, e cioè:

a) aumento di numero delle singole fibre muscolari.

b) costituzione, da parte di esse fibre, di fasci muscolari propriamente detti;

c) diversa direzione di queste fibre e di questi fasci;

d) sviluppo in superficie ed in volume di date zone muscolari, rispetto alla superficie od al volume di tutto il corpo.

\section{Zusammenfassung,}

Mit dem Fortschreiten der aufeinanderfolgenden Beobachtungen, zu deren Vornahme die vorliegende Untersuchung führte, zeigte sich im allgemeinen Kompliziertheit der Muskelmasse bei den Arten mit großem Körpervolumen gegenüber denen mit mittlerem und kleinem. Doch zeigte sich, - gut ansgeprägt in den jeder untersuchten Art speziell beigefügten Zusammenstellungen daß die größere oder geringere Kompliziertheit zuweilen sehr deutlich ist, so daß sich daraus auf einmal eine klare Unterscheidung zweier Typen ergibt, zuweilen aber - wenn sie auch immer durch Rechnung oder Schätzung ermittelbar bleibt - etwas weniger ausgesprochen ist. Es ist aber leicht zu sehen, 
daß wir ans hier auf einem sehr viel ergiebigeren and wechselreicheren Arbeitsgebiete befinden, als ENRIQUES bei seiner Untersuchung der nervösen Ganglien der Wirbellosen. Auch kann man sich leidht von der Schwierigkeit einer Orientierang tiberzeugen, besonders bei einem Gewebe, welches durch seine starke Entwicklung sich als sehr geeignet zu verschiedentlichen Anpassungen und daher auch zu sehr komplizierten Variationen zeigt.

Der charakteristische Bau der Nematoden, bei welchen es möglich ist, die Verschiedenheiten im Bau der kleinen (Meromyarii) und der großen (Polymyarii) durch die Zahl der auf einem Querschnitte des Wurmes vorbandenen Muskelzellen zu definieren, drückt hier in einer der mathematischen Kontrolle zugänglichen Form den ersten von uns gefundenen Komplikationsgrad aus: er wird durch einen numerischen Zuwachs der einzelnen Muskelindividuen repräsentiert.

Bei Sipunculus ist im Vergleich zu Phymosoma die Komplikation etwas weiter fortgeschritten, indem sich die an Zahl gewachsenen Muskelelemente in Gruppen zu zwei, drei und vier Elementen sondern. Bei den großen Pulmonaten und Dibranchiaten zeigen sich die Muskelbündel schon gut individualisiert, wenn man sie dem relativ schlaffen Gewebe der kleinen Formen gegenüberstellt. Bei den großen Oligochaeten endlich machen die Bündel nnd Fasern ganzen Muskelzonen mit scharf ausgesprochenen Grenzen an ihrer Oberfläche Platz, Zonen, die sich bei den Arten mit kleinerer Körpermasse aus der betreffenden Ordnung schlecht begrenzt, weniger ausgedehnt und von schwächlicher und schlaffer Konstitution zeigen.

Ist somit seitens des Muskelsystems das Vorhandensein einer in direktem Verhältnis mit der Artgröße stehende Komplikation der Struktar gesichert, so sind im allgemeinen als verschiedene Modi, unter denen diese Komplikation aich präsentiert, die folgenden aufzastellen:

a) Vermehrung der Zahl der einzelnen Muskelfasern,

b) Konstitation von Muskelbündeln im eigentlichen Sinne seitens dieser Fasern,

c) verschiedene Ausrichtung dieser Fasern und Bündel,

d) Volumen- und Oberflächenentwicklung der sich so ergebenden Muskelzonen nach Maßgabe der Oberfäche und des Volumens des ganzen Körpers.

(Übersetzt von W. Gebhardt.)

\section{VIl. Bibliografia.}

1906. Baвíк, E., Experimentelle Untersuchungen über die Variabilität der Verdauungsröbre. Arch. f. Entw.-Mech. Bd. 21. S. 611-702.

1890. Cerfontaine, P., Recherches sur le système cutané et sur le système musculaire du Lombric terrestre. Arch. Biol. Tom. 10. p. 327.

1868. Claparède, E., Histologische Untersuchungen über den Regenwurm, Lumbricus terrestris L. Zeitschr. f. wiss. Zool. Bd. 19. S. 563.

1909. Dequal, L., Ricerche istologiche sull' epitelio cutaneo e intestinale dell'Octolasium complanatum. Arch. Zool. Vol. IV. Fasc. 2. p. 211-237.

1906. Enriques, P., Della economia di sostanza nelle ossa cave. Arch. f. Entw.-Mech. Bd. 20. S. 427--465.

1907. —- La morte. Riv. di Scienze. Vol. 2. 
1908. Enriques, P., La forma come funzione della grandezza. 2. memoria. Ricerche sui gangli nervosi degli Invertebrati. Arch. f. Entw.-Mech. Bd. 25. S. $655-714$.

1904. Kемma, A., La taille des animanx. Bull. Soc. Zool. malac. Belgique. p. $51-81$.

1906. Levi, G., Studi sulla grandezza delle cellule nei Mammiferi. Arch. ital. di Anat. ed Embr. Vol. 5. Fasc. 2. p. 291-358.

1900. Metalnikoff, S., Sipunculus nudus. Zeitschr. f. wiss. Zool. Bd. 68. S. $262-322$.

1911. Nicodim, H., La topographie des faisceaux musculaires du pied de l'Anodonte. Ann. Scient. de l'Univ. de Jassy. Tom. 7. Fasc. 1. p. 40-52.

Ribaucourt, E., Étude sur l'anatomie comparée des Lombricides. Bull. Sc. Franc. Belg. Tom. 35. p. 211.

1881. Roux, W., Der Kampf der Teile im Organismus. Leipzig.

1907. - Über die funktionelle Anpassung des Muskelmagens der Gans. Arch. f. Entw.-Mech. Bd. 21. S. 461-499.

\section{Spiegazione delle figure.}

\section{Tavola XII.}

Fig. 1. Fasciola hepatica. Sezione sagittale e apicale di un grosso individuo Oc. 2, 0b. 4, alz. 16. $s v$ sezione di parte della ventosa orale (sono disegnate solamente le fibre radiali); $s v^{1}$ sezione dell' altra parte della ventosa orale (sono disegnate le fibre meridiane, quelle trasverse e quelle equatoriali; queste ultime rappresentate dai punti neri); sf faringe (le fibre radiali evidentissime, le circolari indicati dai punti); $m p$ muscolo protrattore della faringe; $a i$ ansa intestinale. Il resto è trascurato.

Fig. 2. Fasciola hepatica. Schematica per determinare l'andamento delle fibre dei tre strati muscolari costituenti il mantello che avvolge il corpo dell'animale.

Fig. 3. Dicrocoelium lanceatum. Schema della sezione saggittale ed apicale. E visibile la ventosa e la faringe.

Fig. 4. Eiseniella tetraedra. Sezione trasversa del corpo nella sua parte media. Del terzo strato muscolare è solo accennato il contorno. Oc. 2, 0b. 4, alz. 16. $z d$ zone dorsali dei muscoli del $3^{\circ}$ strato; $z v$ zona ventrale; $z l$ zone laterali; $z a$ zone accessorie; $s$ setole.

Fig. 5. Octolasium complanatum. Porzione di sezione trasversa del corpo, nella sua parte media. Del terzo strato sono state solamente disegnate le lamelle di sostegno; nel secondo è stato trascurato il pigmento. Oc. 2, Ob. 4, alz. 16 .

Fig. 6. Eiseniella tetraedra. Sezione sagittale del corpo. La linea nera dopo lepidermide rappresenta il secondo strato di muscoli circolari. Il terzo strato striato è quello dei muscoli pennati. Nel mezzo la massa intestinale. 0 c. 2 , 0b. 4 , alz. 16.

Fig. 7. Sipunculus nudus. Sezione sagittale della massa costituita dai muscoli retrattori della proboscide. Le linee rappresentano i limiti dei singoli fasei. 0c. 3 , Ob. 6, alz. 16.

Fig. 8. Sipunculus nudus. Sezione trasversale dei medesimi muscoli. Oc. 3, 0b. 6, alz. 16 . 
Fig. 9. Phymosoma granulatum. Sezione sagittale dei muscoli della proboscide. Oc. 3, 0b. 6, alz. 16.

Fig. 10. Phymosoma granulatum. Sezione trasversa dei medesimi muscoli. Oc. 3, 0b. 6, alz. 16.

Fig. 11. Sepiola rondeletii. Parte della sezione trasversa di un braccio. I soli contorni.

Fig. 12. Loligo vulgaris. Parte della sezione trasversa di un braccio. Schematica. Nel primo strato sono ommesse le fibre longitudinali. Il secondo strato, nero, rappresenta la zona a fibre circolari. Del terzo sono visibili solo le zone interposte alle sezioni trasverse di fibre, le quali ultime occuperebbero, di conseguenza, tutta la parte bianca. Del quarto sono accennati i soli contorni.

Fig. 13. Octopus De-Filippii. Parte della sezione trasversa di un braccio. I punti neri indicano $i$ tagli trasversi delle fibre. Oc. 2,0 b. 4, alz. 16.

Fig. 14. Helix adspersa. Sezione trasversale della faringe. Zona a fascetti di fibre. Qui i punti neri indicano le sezioni trasverse delle fibre.

Fig. 15. Eledone muscata. Parte della sezione trasversa di un braccio. I piccoli cerchi indicano le sezioni delle fibre trasverse. Oc. 2, 0b. 4, alz. 16.

\section{Tavola XIII.}

Fig. 16. Helix adspersa. Sezione trasversale della faringe. Zona di fasci a struttura compattissima. I cerchietti indicano piccole zone di fibre tagliate trasversalmente; $i$ punti neri indicano $i$ nuclei. Oc. 2, Ob. 6, alz. 16.

Fig. 17. Helix lucorum. Sezione della massa muscolare del piede. Zona con fibre riunite in fasci grossolani. Oc. 2, Ob. 6, alz. 16.

Fig. 18. Helix nemoralis. Sezione trasversale della faringe. Zona a fibre isolate ed incrociate. I punti neri indicano le solite sezioni trasverse delle fibre. Oc. 2, Ob. 6, alz. 16.

Fig. 19. Helix adspersa. Taglio interessante il piede e la faringe. Zona a piccoli fascetti ed a fibre isolate. Oc. 2,0 b. 6, alz. 16 .

Fig. 20. Helix nemoralis. Taglio interessante, come il precedente, il piede e la faringe. Zona a fibre pressate ed orientate in un sol verso. 0c. 2,0 b. 8 , alz. 16.

Fig. 21. Helix variabilis. Sezione della massa muscolare del piede. Zona a fibre isolate e poliorientate. 0 c. 2,0 b. 6 , alz. 16. 

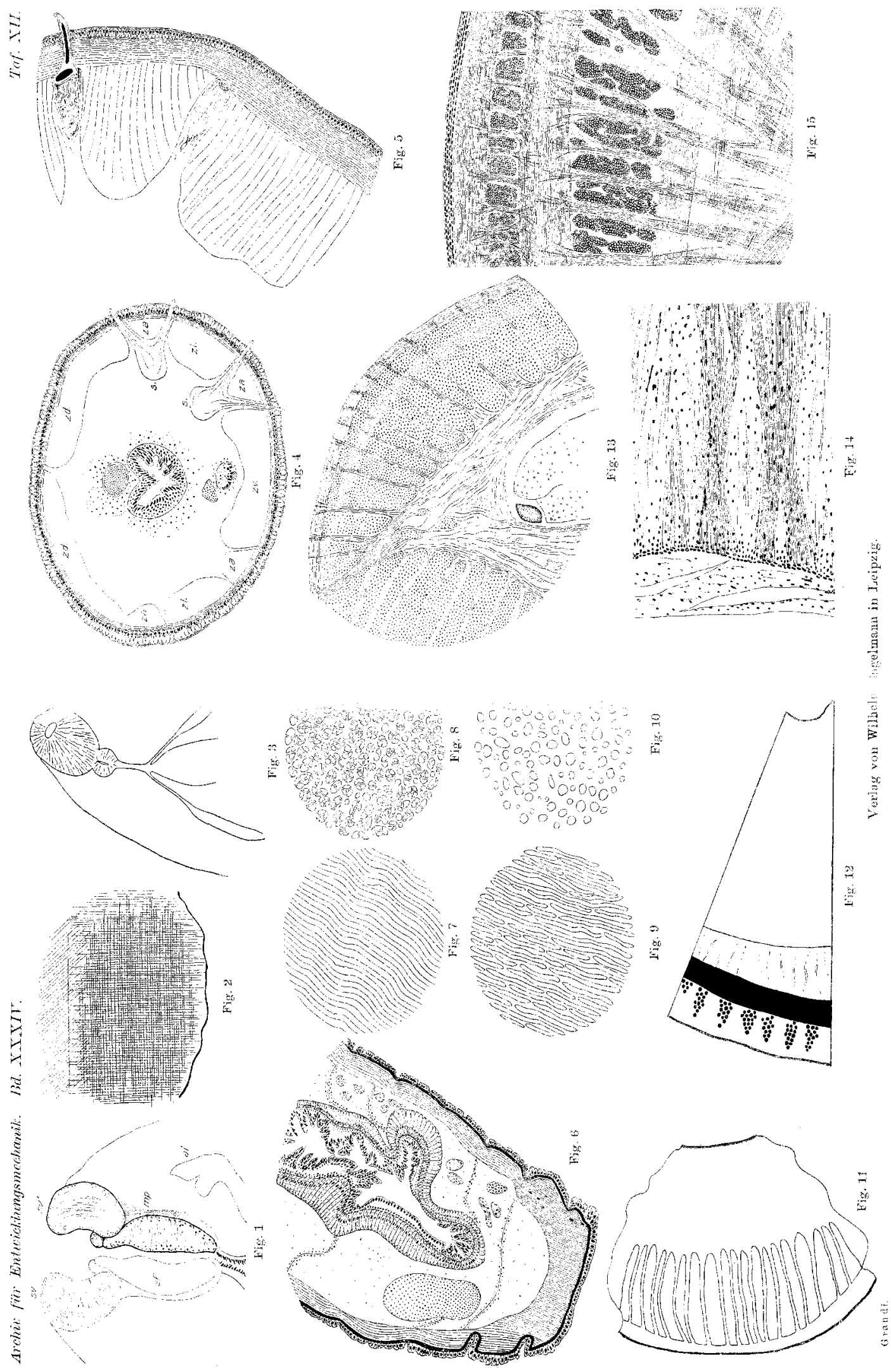

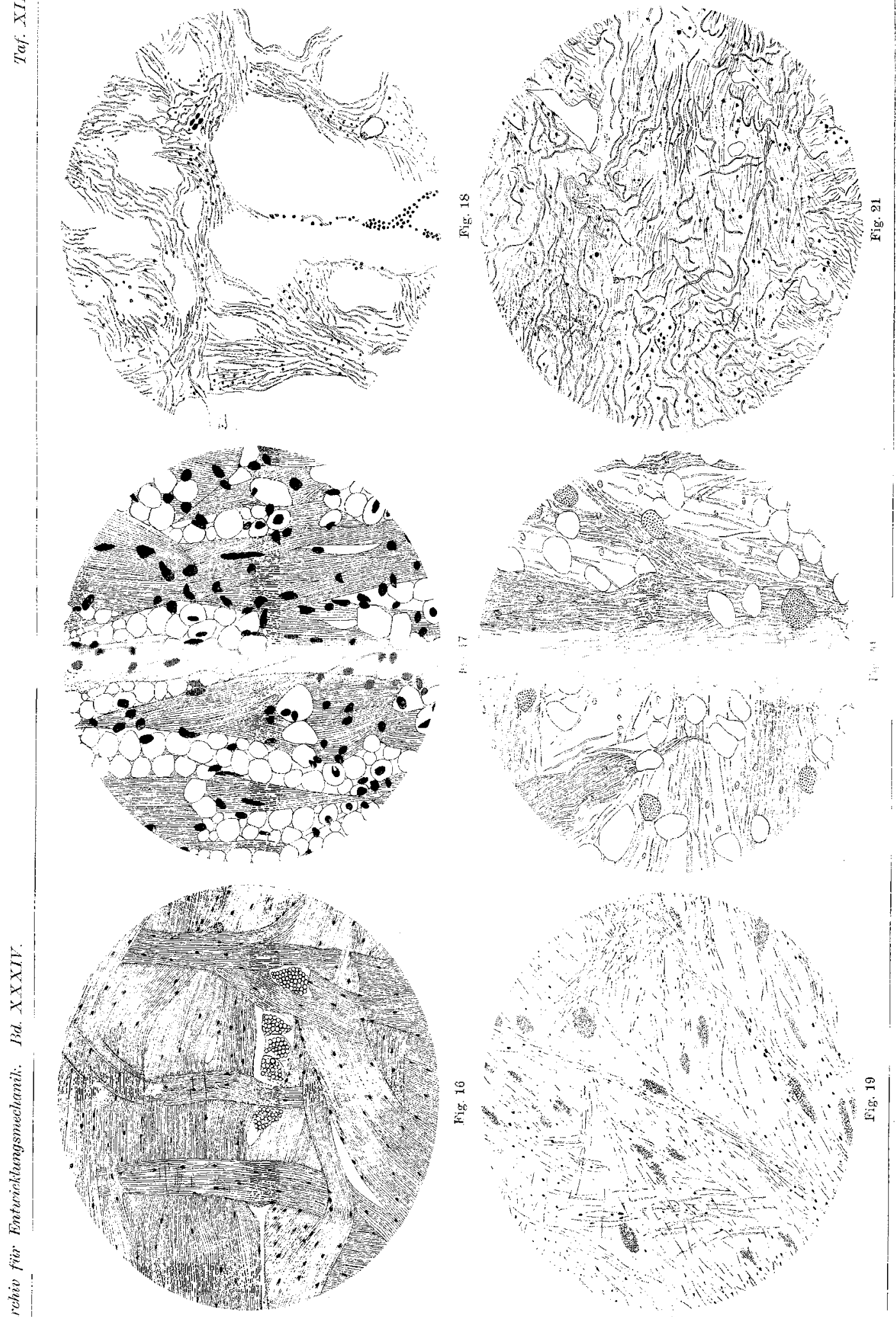